\title{
Cost-effective DWDM ROADM Design for Flexible Sustainable Optical Metro-Access Networks
}

\author{
Samael Sarmiento, Jose A. Altabas, David Izquierdo, Ignacio Garces, Salvatore Spadaro, \\ and Jose A. Lazaro
}

\begin{abstract}
Architectural changes are required at the underlying networks to support the expected Internet data traffic volume growth caused by the popularization of cloud services, 5G-based services, and social networks, whereas providing a highly dynamic connectivity. Cost-effective and energy efficient solutions for flexible network subsystems are required in order to provide future sustainable networks. In this paper, we present a cost-effective DWDM ROADM design enabling optical Metro-Access networks convergence. The cost-effective DWDM ROADM capabilities have been also assessed in an ultra-Dense Wavelength Multiplexing (u-DWDM) Ring Network scenario. In particular, the achievable network throughput has been considered.
\end{abstract}

\section{Index Terms-DWDM; ROADM; Throughput.}

\section{INTRODUCTION}

$\mathbf{T}$ The ICT eco-system has been rapidly and dramatically changing in the last years. New multimedia and cloud services, the deployment of the Internet of Things (IoT) and the convergence between optical and wireless communications at the $5 \mathrm{G}$ paradigm [1] are requiring changes to the networks in order to enable scalable growth in traffic volume, while supporting a high level of dynamic connectivity, full flexibility and increased energy-efficiency. These features can be achieved by considering the cooperation between the network control and data planes. On the one hand, the management and control of networks are evolving towards a Software Defined Networking (SDN)-based centralized architecture (see Fig. 1). On the other hand, Metro Networks are converging with Access Networks and evolving towards all-optical solutions [2, 3]. In this context, transmission techniques based on ultraDense Wavelength Multiplexing (u-DWDM) are a promising alternative to Time Division Multiplexing (TDM) solutions due to their high spectral efficiency [4], which is

Manuscript received May 09, 2017.

Samael Sarmiento (samael.sarmiento@tsc.upc.edu), Jose A. Altabás, David Izquierdo, Ignacio Garcés, Salvatore Spadaro, and Jose A. Lazaro are with Universidad de Zaragoza (UNIZAR), Centro Universitario de la Defensa de Zaragoza, Universitat Politècnica de Catalunya (UPC) - Barcelona Tech, 08034 Barcelona, Spain. accomplished by the subdivision of a DWDM channel into smaller channels called frequency slots (FS) wherein the uplink (U) and the down-link (D) for each user can be established (see Fig. 1).

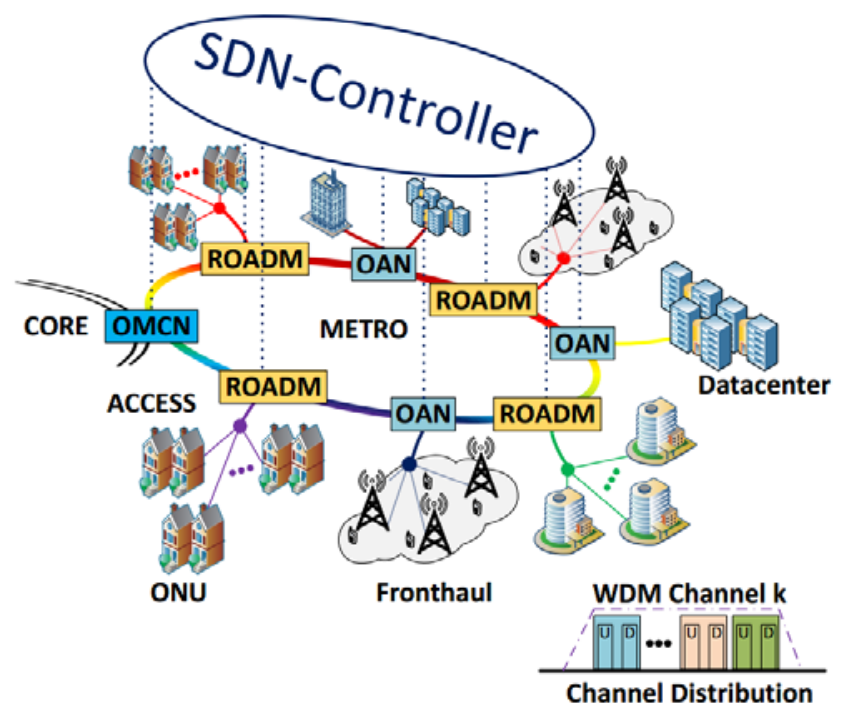

Fig. 1. Flexible 5G Metro-Access Network scenario. Inlet: proposed flexible ultra-dense WDM full-duplex frequency slot division.

This paper is divided into five sections. Section II presents the proposed cost-effective DWDM Reconfigurable Optical Add-Drop Multiplexer (ROADM) node design, its operation modes and the main advantages of the proposed ROADM architecture. Section III presents the insertion loss, sensitivity and crosstalk measurements and their experimental set-up used for the ROADM characterization. Section IV presents the considered u-DWDM network scenario for proving the cost-effective DWDM ROADM capabilities; an iterative process to design each costeffective DWDM ROADM is proposed in order to obtain the achievable network throughput. Finally, Section V completes the paper with the main conclusions.

\section{DWDM ROADM NODE ARCHITECTURE}

The ROADM design for future Metro-Access converged Networks is basically driven by new network-level requirements, such as full flexibility, adaptability, scalability, resilience and increased energy-efficiency [5]. In order to reach all those features, a new cost-effective 
DWDM ROADM node design is here proposed.

In line with the scenario shown in Fig. 1, the MetroAccess Network relies on different node elements. In particular, the ROADMs are all-optical nodes that connect the Metro to the Access Networks [6]. The Optical MetroCore Node (OMCN), the Optical Aggregation Node (OAN) and the Optical Network Unit (ONU) still perform OpticalElectrical-Optical (OEO) conversion for their functions. Specifically, the OMCN provides transfer of information between the Metro and Core Network; the OAN is the network element to provide connectivity between the Metro Network and Datacenters or 5G-Fronthault Networks; and the ONU between the Access Network and the final Users.

Figure 2 shows an example of architecture of a bidirectional ROADM with colorless and directionless (CD) features [7-9] that can be used in the proposed scenario shown in Fig. 1 in which both Metro Network and Access Network transmission systems are based on single bidirectional fiber links and the considered multiplexing technique is u-DWDM, characterized by dividing each WDM channel into smaller channels called frequency slots (FS) [10]. The ROADM is based on three $1 \times 2$ WavelengthSelective Switches (WSSs) which allow implementing add/drop and pass-through functions for each channel individually. In this way, when add/drop function is selected for a specific channel, it can be routed to/from the West (W) or East (E) ports in the metro part of the network from/to the Add/Drop (A/D) port where the access part of the network is connected. On the other hand, when the passthrough function is chosen, the selected channel can be routed from/to W/E port to/from E/W port. This architecture can be implemented to work with a frequency slot granularity of $12.5 \mathrm{GHz}$ using nowadays commercial WSSs $[11,12]$, and it is expected to supports $6.25 \mathrm{GHz}$ granularity and even lower in the near future. This architecture providing a high performance colorless and directionless functionality at the FS level, though at relatively high cost due to the WSS modules [13], is the reference architecture for comparison in this paper. In order to reach a trade-off solution between performance and costs, a cost-effective DWDM ROADM architecture is proposed.

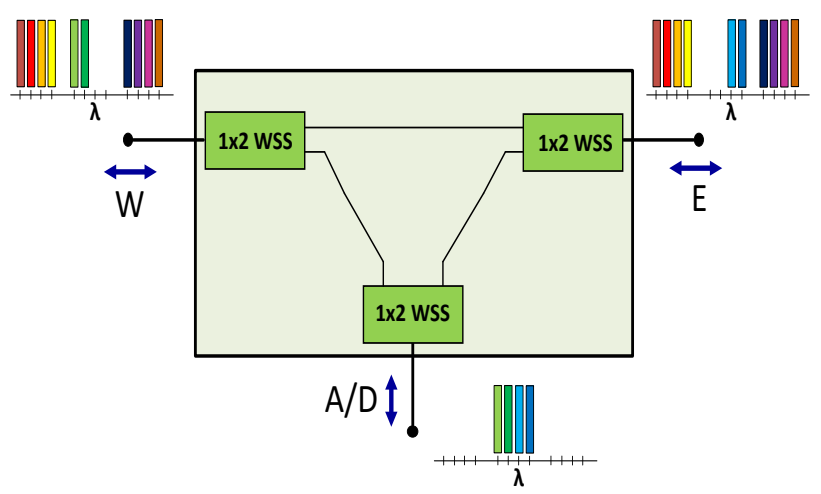

Fig. 2. Reference colorless and directionless ROADM set-up.

Figure 3a shows the basic stage of the bidirectional costeffective ROADM that can switch a group of frequency slots of the same WDM channel. The basic stage of the ROADM $\left(B W_{i}\right)$ consists of two fixed filters and two latched switches.
The filtered WDM channel may pass through the module or can be added/dropped from/to the Access Network depending on the $2 \times 2$ switch configuration. The $1 \times 2$ switch determines over which port, $\mathrm{E}_{\mathrm{i}}$ or $\mathrm{W}_{\mathrm{i}}$, the connection is established. This ROADM design can be scaled to manage other wavelengths through the addition of similar stages to that shown in Fig. 3a as it is indicated in Fig. 3b, where a 1 to $\mathrm{N}$ power splitter $(1: N)$ can be used for this purpose, thus reducing the cost of the proposed ROADM. However, the losses of the ROADM increases significantly when the number of stages of the ROADM $(N)$ improves due to the splitter. In this way, an optical insertion losses (IL) study of the cost-effective ROADM depending on the $N$ must be made. Section III shall provide that study. In addition, for cases where the number of stages could be high, a wavelength division multiplexer (WDM) can be used instead of the power splitter for reducing losses. Therefore, it enables the coverage of both $\mathrm{C}$ and $\mathrm{L}$ bands. In that case, the cost-effective ROADM works as a CD all-optical node at DWDM channel level [7-9], while the WSS-based reference ROADM, Fig. 2, is a CD ROADM node at FS level.

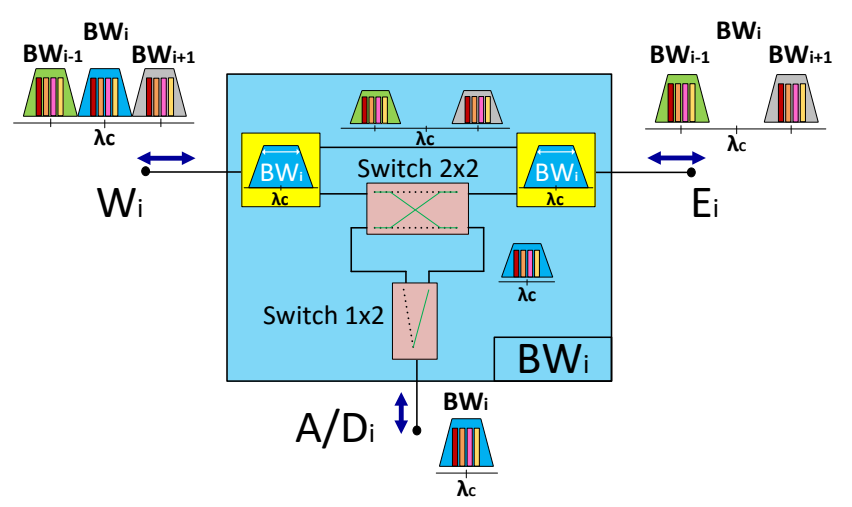

(a)

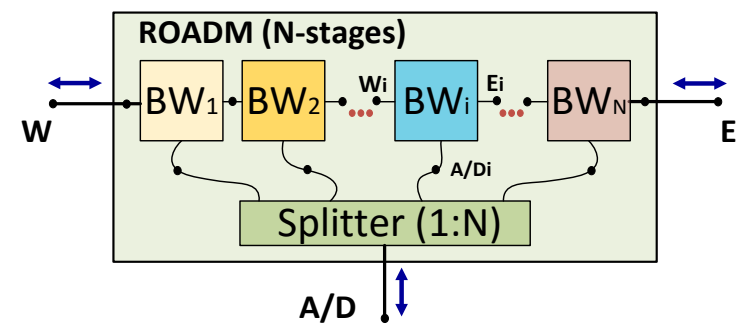

(b)

Fig. 3. a) Set-up of a basic stage of the cost-effective ROADM; b) Cost-effective ROADM design for $\mathrm{N}$ stages.

Taking into account the cost-effective ROADM node architecture, the major advantages that carrier companies can enjoy are:

- Off-the-shelf Components. The proposed cost-effective ROADM, Fig. 3, consists mainly of three-port fixed filters and latched switches $(1 \times 2$ and $2 \times 2)$. On the one hand, there is a huge choice of switches in the niche market that can operate over $\mathrm{C}$ and $\mathrm{L}$ bands at lower cost $[14,15]$. On the other hand, the prize of the fixed filters depends on their bandwidth, being highly available those whose bandwidth is between $2 \mathrm{THz}$ and 
$50 \mathrm{GHz}[14,15]$. Therefore, the proposed cost-effective ROADM can be easily adapted to work width CWDM or DWDM channels relying upon the requirements of vendors. For lower ROADM granularity, it is necessary to change the used technology to Arrayed Waveguide Grating (AWG) or WSS. These technologies improve the implementation cost of the proposed ROADM [11, 12, $16,17]$, but obtaining a bandwidth of $12.5 \mathrm{GHz}$.

- Reduced Cost. The cost-effective ROADM is based on the so called First Generation Type-I ROADM [18, 19]. They employ a demux-switch-mux approach to add/drop/pass-through that is characterized by presenting low cost, scalable implementation. Tables I and II allow to benchmark the cost implementation of the WSS-based ROADM and the cost-effective ROADM, respectively. In particular, Table I shows the unit cost values (in arbitraries units) of different components required for both architectures. Those values have been normalized to the cost of a $200 \mathrm{GHz}$ spacing single channel DWDM multiplexer, e.g. DWDM-200-47 whose price is $175 \$$ as it is indicated in [20]. Table II shows the required components number and the total unit cost (TUC) for: a) a WSS-based reference ROADM node at FS level, Fig. 2; b) a WSS-based ROADM node at DWDM channel level; and c) the proposed cost-effective ROADM design, Fig. 3; all of them covering the whole C band. So, for the WSS-based ROADM, two different granularities have been considered. The first one is $12.5 \mathrm{GHz}$, which corresponds with the considered frequency slot granularity. The second one is $100 \mathrm{GHz}$ that is a granularity close to the proposed cost-effective ROADM of $125 \mathrm{GHz}$. The choice of that value for the cost-effective ROADM granularity shall be demonstrated in Section IV. Due to the fact that for both ROADM's architectures all the components are offthe-shelf, factors such as the maturity, production volume and components' performance have been taken into account to calculate the TUCs of the different ROADM implementations proposed. Finally, the considered configuration of the proposed cost-effective ROADM design covering a whole band, with $\mathrm{N}=20$, Fig. $3 b$, can be considered the worst case, while in general $1 \leq \mathrm{N} \leq 20$, and for many cases a single stage ROADM, $\mathrm{N}=1$, as in Fig. 3a is enough, as it shall be seen in an example in Section IV. From Table II, we can notice that the proposed cost-effective DWDM ROADM total unit cost is, for the case of $\mathrm{N}=20(\mathrm{~N}=1), 10.6(190.2)$ times lower than the total unit cost of the WSS-based ROADM with a FS granularity of $12.5 \mathrm{GHz}$ and 8.1(146.3) times lower than $100 \mathrm{GHz}$ granularity.

TABLE I

NORMALIZED UNIT COST FOR PROPOSED COMPONENTS

\begin{tabular}{|c|c|c|}
\hline \hline Component & $\begin{array}{c}\text { Normalized unit } \\
\text { cost }\end{array}$ & Refs. \\
\hline $1 \times 32$ splitter & 0.34 & {$[21]$} \\
\hline Dual 1x2 SW & 0.40 & {$[22]$} \\
\hline Dual $2 \times 2$ SW & 0.54 & {$[23]$} \\
\hline $\begin{array}{c}\text { 1x2 WDM mux } \\
(175 \mathrm{GHz})\end{array}$ & 1 & {$[20]$} \\
\hline
\end{tabular}

\begin{tabular}{|c|c|c|}
\hline $\begin{array}{c}\text { 1x2 WSS } \\
(12.5 \mathrm{GHz})\end{array}$ & 208 & {$[11,13]$} \\
\hline $1 \times 2$ WSS $(100 \mathrm{GHz})$ & 160 & {$[11]$} \\
\hline \hline
\end{tabular}

TABLE II

COMPONENTS NUMBER LIST FOR THE WSS-BASED ROADM FOR DIFFERENT GRANULARITIES AND THE COST-EFFECTIVE ROADM, AND THEIR TOTAL UNIT COST

\begin{tabular}{|c|c|c|c|c|c|c|c|c|}
\hline \multirow[b]{2}{*}{ 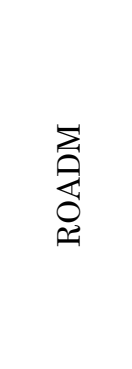 } & \multirow[b]{2}{*}{ 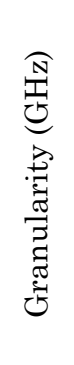 } & \multicolumn{6}{|c|}{ Number of Components } & \multirow[b]{2}{*}{ 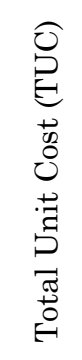 } \\
\hline & & 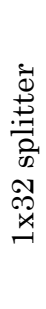 & 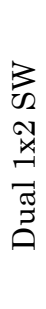 & 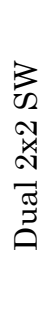 & 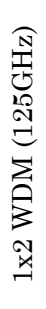 & 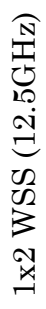 & 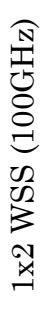 & \\
\hline \multirow{2}{*}{$\begin{array}{l}\text { WSS- } \\
\text { based }\end{array}$} & 12.5 & & & & & 3 & & 624 \\
\hline & 100 & & & & & & 3 & 480 \\
\hline $\begin{array}{c}\text { Cost- } \\
\text { effective }\end{array}$ & 125 & 1 & 20 & 20 & 40 & & & 59.14 \\
\hline
\end{tabular}

- Pay-as-You-Grown. Cost-effective ROADM is an excellent low cost solution for growing metro-access networks. If the number of demands increases, the number of channels can be increased, as one stage handles one channel. Adding one new channel at a time allows on-demand service introduction with minimal initial investment. However, the proposed architecture of the cost-effective ROADM shown in Fig. 3 has to be upgraded so as to avoid that the ongoing traffic could be interrupted because of adding new stages.

- Low Commutation Time. The commutation time of the proposed cost-effective ROADM only depends on the commutation speed of a switch. In that case, the potentially switching speed of the proposed costeffective ROADM is lower than $8 \mathrm{~ms}[22,23]$. That value is within the same order of magnitude of the switching speed of the WSS-based ROADM whose switching speed is of a few milliseconds [11, 12, 24].

- Reduced Power Consumption. Table III shows the worst case of power consumption of both WSS-based and costeffective ROADMs when they work for whole $\mathrm{C}$ band. To the cost-effective ROADM, the worst case power consumption corresponds to $N=20$ when it is considered that each ROADM's stage present an effective bandwidth of $125 \mathrm{GHz}$. From Table III, it can notice that the power consumption of the cost-effective ROADM is 3.5 times lower than the WSS-based ROADM. That node power consumption reduction is because of the proposed cost-effective ROADM uses latched switches $[22,23]$ which maintain its state after being commuted, allowing keeping the power consumption reduced. This make that the cost-effective ROADM can be a good solution for future sustainable networks. Furthermore, cost-effective ROADM reduces the instances of OEO conversions by setting all-optical links between metro and access nodes. That helps to reduce the power consumption of the network. 
TABLE III

POWER CONSUMPTION OF THE WSS-BASED AND COST-EFFECTIVE ROADMS

\begin{tabular}{|c|c|c|}
\hline \hline ROADM & $\begin{array}{c}\text { WSS-based } \\
{[11]}\end{array}$ & $\begin{array}{c}\text { Cost-effective } \\
{[22,23]}\end{array}$ \\
\hline $\begin{array}{c}\text { Power } \\
\text { Consumption (W) }\end{array}$ & 25.2 & 7.2 \\
\hline
\end{tabular}

- Coexistent with legacy systems. The proposed granularity for the cost-effective ROADM permits that it can be compatible with various technology transmission systems such as NGPON2 which required channels with $100 \mathrm{GHz}$ of bandwidth [25] and u-DWDM [10].

\section{COST-EFFECTIVE ROADM EXPERIMENTAL CHARACTERIZATION}

In order to characterize the basic stage of the proposed cost-effective ROADM, three types of measurements have been realized: i) spectral characterization which permits to determine the effective bandwidth, the insertion loss (IL) and the isolation among ROADM ports; ii) single channel data transmission sensitivity for checking potential distortions in the received signal; and iii) multiple channel data transmission sensitivity for checking potential crosstalk due to lack of full isolation between $\mathrm{W}, \mathrm{E}$ and $\mathrm{A} / \mathrm{D}$ ports. All these measurements have been done for a single stage ROADM based on two fixed filters with a bandwidth of $175 \mathrm{GHz}$ and centered at $1539.77 \mathrm{~nm}$ (JDS Uniphase [20]), and two commercial opto-mechanical latched switches [22, 23] controlled by a prototype current source and a micro-PC.

Figure 4 shows the ROADM effective bandwidth (at $-3 \mathrm{~dB}$ cut-off frequencies), $\mathrm{BW}=125 \mathrm{GHz}$, and its $37.5 \mathrm{GHz}$ guard bands (GB). The out-band and in-band insertion losses for the pass-through configuration (PT IL line in Fig. 4, measured $\mathrm{W} \leftrightarrow \mathrm{E}$ and switch $2 \times 2$ in Fig. $3 \mathrm{a}$ in bar state) are: $\mathrm{IL}_{\mathrm{o}-\text { band }}^{\mathrm{PT}}=0.6 \mathrm{~dB}$ and $\mathrm{IL}_{\mathrm{i}-\mathrm{band}, \mathrm{PT}}^{\mathrm{PT}}=2 \mathrm{~dB}$, respectively. And the inband IL for the add/drop configuration (A/D IL line in Fig. 4, measured from $\mathrm{W} / \mathrm{E} \leftrightarrow \mathrm{A} / \mathrm{D}$ and switch $2 \times 2$ in Fig. 3a in cross state) is $I L_{i-b a n d, A / D}^{A}=2 d B$. The in-band add/drop isolation, equivalent to an IL between pass-through ports, but when the channel is added/dropped, is $\mathrm{IL}_{\mathrm{i}-\text { band, } \mathrm{A} / \mathrm{D}}^{\mathrm{PT}}=35 \mathrm{~dB}(\mathrm{~A} / \mathrm{D}$ isolation line, measured $\mathrm{W} \leftrightarrow \mathrm{E}$ and switch $2 \times 2$ in Fig. $3 \mathrm{a}$ in cross state) On the other hand, the in-band pass-through isolation, equivalent to an IL between $\mathrm{W} / \mathrm{E}$ and $\mathrm{A} / \mathrm{D}$ ports when the channel pass-through the stage, is $\mathrm{IL}_{\mathrm{i}-\text { band,PT }}^{\mathrm{A} / \mathrm{D}} \mathrm{P}$ $60 \mathrm{~dB}$ (PT isolation line, measured $\mathrm{W} / \mathrm{E} \leftrightarrow \mathrm{A} / \mathrm{D}$ and switch $2 \times 2$ in Fig. 3a in bar state). Those measurements were obtained with a BOSA High Resolution Optical Complex Spectrum Analyzer (HROCSA) from Aragon Photonics Labs.

Once characterized the insertion losses of a single ROADM stage, the optical insertion loss (IL) for a ROADM based on $\mathrm{N}$ stages can be calculated as it is indicated in Eq. (1-4), where $\mathrm{i}, \mathrm{N}$ and $\mathrm{BW}_{\mathrm{i}}$ are the stage position in the ROADM, the number of ROADM stages, and the range of wavelengths inside the effective bandwidth of the i-stage, respectively. Equation (1) represents the ROADM insertion loss between $\mathrm{W}$ and $\mathrm{E}$ ports, expressed in $\mathrm{dB}$, for wavelengths out of the range of operation of all ROADM stages, $\lambda \notin B W_{i}(1 \leq i \leq N), I L_{\lambda \notin B W_{i}}^{W \leftrightarrow E}$, while in Eq. (2) is indicated the losses between $\mathrm{W}$ and $\mathrm{E}$ ports for wavelengths in the range of operation of the ROADM i-stage $(1 \leq i \leq N)$, $I L_{\lambda \in B W_{i}}^{W \leftrightarrow E}$ in PT configuration. As it can be seen in both $I L_{\lambda \notin B W_{i}}^{W \leftrightarrow E}$ and $I L_{\lambda \in B W_{i}}^{W \leftrightarrow E}$ equations, they scale linearly with the number stages of the ROADM. Equations (3) and (4) express the insertion loss between $\mathrm{W}$ and $\mathrm{A} / \mathrm{D}$ ports and $\mathrm{E}$ and $\mathrm{A} / \mathrm{D}$ ports, respectively, for the add/drop configuration. In both cases, the third term includes the insertion loss of a real 1 to $\mathrm{N}$ power splitter.

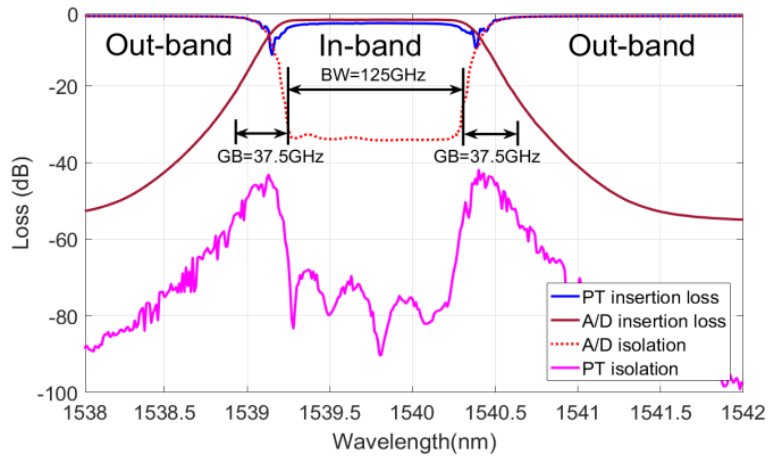

Fig. 4. Single stage cost-effective ROADM IL for pass-through (PT) and add/drop (A/D); and isolation: add/drop (A/D) and pass-through (PT).

$$
\begin{gathered}
I L_{\lambda \notin B W_{i}}^{W \leftrightarrow E}(d B)=I L_{o-\text { band }}^{P T} * N=0.6 * N \\
I L_{\lambda \in B W_{i}}^{W \leftrightarrow E}(d B)=I L_{o-\text { band }}^{P T} *(N-1)+I L_{i-b a n d, P T}^{P T} \\
=0.6 *(N-1)+2 \\
I L_{\lambda \in B W_{i}}^{W \leftrightarrow A / D}(d B)=I L_{o-\text { band }}^{P T} *(i-1)+I L_{i-\text { band }, \mathrm{A} / \mathrm{D}}^{A / D} \\
+3.5 * \operatorname{ceil}\left(\log _{2}(N)\right) \\
=0.6 *(i-1)+2+3.5 * \operatorname{ceil}\left(\log _{2}(N)\right) \\
I L_{\lambda \in B W_{i}}^{E \leftrightarrow A}(d B)=I L_{o-\text { band }}^{P T} *(N-i)+I L_{i-\text { band }, \mathrm{A} / \mathrm{D}}^{A / D} \\
+3.5 * \operatorname{ceil}\left(\log _{2}(N)\right) \\
=0.6 *(N-i)+2+3.5 * \operatorname{ceil}\left(\log _{2}(N)\right)
\end{gathered}
$$

As commented, also a WDM can be used instead of the power splitter for reducing losses in case of high $\mathrm{N}$. Therefore, considering a 1 to $\mathrm{N}$ power splitter covers the worst case for next section network analysis. Taking into account Eq. 1-4, the worst case insertion loss $\left(I L_{W C}\right)$ of the cost-effective ROADM working for whole $\mathrm{C}$ band, $N=20$ using $200 \mathrm{GHz}$ spaced filters, is $30.9 \mathrm{~dB}[22,23]$ while the WSS-based ROADM is of $10 \mathrm{~dB}$ [11]. Nevertheless, the $I L_{W C}$ of cost-effective ROADM can be reduced to $18.4 \mathrm{~dB}$ when a WDM [26] is used instead of the power splitter. Furthermore, in many cases, a single stage cost-effective $\operatorname{ROADM}(N=1$, Fig. 3 ) could be enough to provide service to the user of a PON, as it shall be demonstrate in one network scenario example in Section IV. In that case, the $I L_{W C}$ of the cost-effective ROADM is just of $2 \mathrm{~dB}$.

The experimental set-up for sensitivity and crosstalk measurements are shown in Fig. 5. The transmitter (TX) is based on an external cavity, $100 \mathrm{kHz}$ linewidth, Tunable Laser Source (TLS), modulated by a Mach-Zehnder Modulator (MZM). The TLS is used to adjust its wavelength inside the frequency slot for these measurements. The MZM is set at the minimum transmission point of the MZM for 
phase modulation and it is thermally controlled to ensure its stability. The optical transmitter uses a Digital Transmitter (DTX) unit, where the data is differentially encoded and shaped to achieve maximum performance for a $1 \mathrm{Gbps}$ data-stream. The transmitted symbols in the Digital Transmitter (DTX) are filtered using a Nyquist Pulse Shaper filter with 12 -symbols filter length and zero roll-off factor. The DTX is implemented in MATLAB ${ }^{\mathrm{TM}}$ and the electrical signals are generated by a $12 \mathrm{GSa} / \mathrm{s}$ Arbitrary Waveform Generator [10, 27].

The receiver $(\mathrm{RX})$ is based on a single photodetector heterodyne detection. The Local Oscillator (LO) is also an external cavity TLS. In the proposed set-up, the LO and the signal are coupled at the photodiode using an optical coupler (DC) and a polarization controller though this heterodyne receiver can be easily upgradeable to a polarization insensible heterodyne receiver as it is indicated in [28]. After the heterodyne detector, the received signal has been optically down-converted to an Intermediate Frequency (IF) equal to $2 \mathrm{GHz}$. The IF signal passes through a DC-Block and it is digitalized with a 40GSa/s Digital Oscilloscope [10, 27]. The first step in the digital processing is the bandpass filtering of the digitalized signal with a FIR filter in order to reduce the noise. Then, to demodulate the DPSK format, the signal is multiplied with itself delayed one symbol and lowpass filtered with a FIR filter. Finally, the BER is calculated comparing the detected data-stream with the original one. A Variable Optical Attenuator (VOA) has been used to modify the received power.

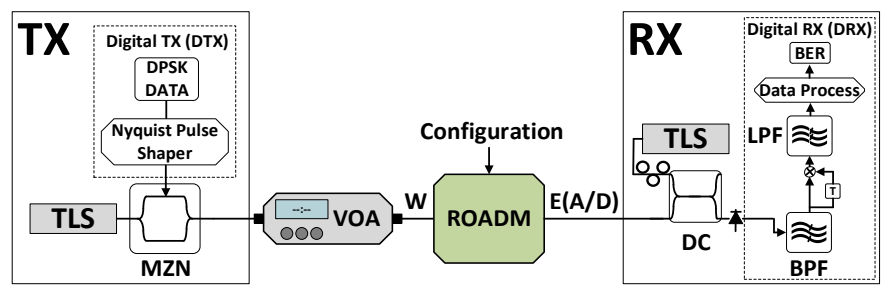

(a)

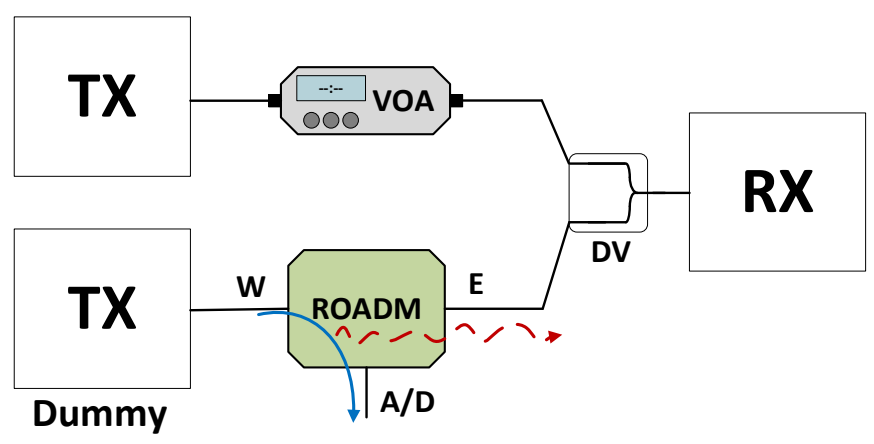

(b)

Fig. 5. Experimental set-up for sensitivity (a) and crosstalk (b) measurements.

The sensitivity, defined as the minimum received power to ensure a BER of $2.2 \cdot 10^{-3}$ without FEC and $10^{-12}$ with a $7 \%$ overhead FEC [29], has been evaluated for a $1 \mathrm{Gbps}$ data-stream over a $6.25 \mathrm{GHz}$ Frequency Slot (FS). Nevertheless the proposed network scenario considers that the down-link (D) and up-link (U) for a user are in the same FS. It has been demonstrated in [10] that the penalty when both $\mathrm{U}$ and $\mathrm{D}$ are established in the same FS is negligible for a similar transmission as the system shown in Fig. 5a. Therefore, any possible penalty in the sensitivity is just due to the proposed ROADM and it can be evaluated using only one channel in the FS.

The sensitivity measurement is taken in five different cases. The sensitivity for back-to-back (BTB) transmission is $-48 \mathrm{dBm}$, as shown in Fig. 6 . No sensitivity penalties were observed for a single channel located out-band and in-band for pass-through configuration and also for in-band for the add/drop configuration, at the single stage ROADM characterized in Fig. 4. Hence, neither the used latched switches nor the band filters do add any observable penalties.

In order to measure the impact of the limited passthrough isolation between $\mathrm{W}$ and E ports (35dB, Fig. 4), the following crosstalk measurement has been done (Fig. 5b): a dummy $1 \mathrm{Gbps}$ data-stream over a $6.25 \mathrm{GHz}$ frequency slot (FS) is dropped at the ROADM A/D port, while the BER of another similar $1 \mathrm{Gbps}$ channel at the same wavelength is measured. For a dummy channel received power of $-38 \mathrm{dBm}$, the crosstalk penalty is $1.75 \mathrm{~dB}$ as shown in Fig. 7. That crosstalk penalty value is reduced as the number of ROADM stages increases.

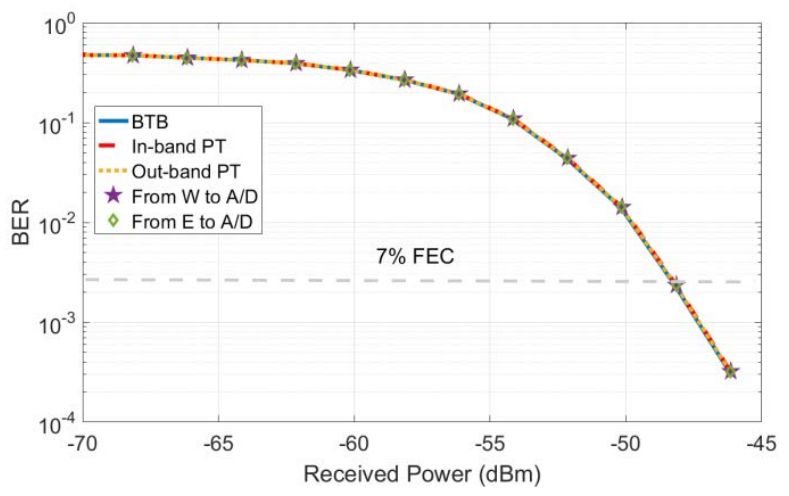

Fig. 6. BER versus received power for a $1 \mathrm{Gbps}$ over a $6.25 \mathrm{GHz}$ frequency slot for back-to-back (BTB), pass-through (PT) and add/drop (A/D) configurations.

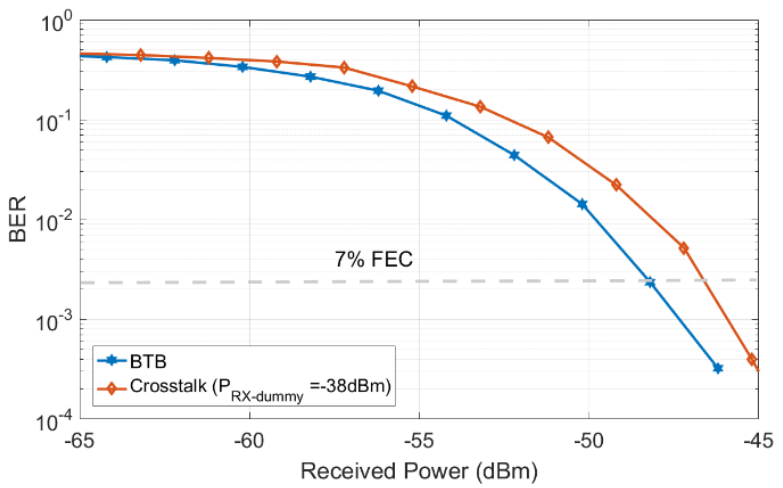

Fig. 7. BER versus received power for a $1 \mathrm{Gbps}$ data-stream over a $6.25 \mathrm{GHz}$ frequency slot when a similar channel is dropped at the ROADM A/D port.

\section{Metro Ring Network BAsed On COST- EFFECTIVE DWDM ROADMs}

On the basis of the characterization performed in the 
previous section, we assess now the performance of the proposed cost-effective DWDM ROADM as a Metro-Access Ring Network element; the assessment is performed in terms of the maximum throughput that the network can achieve taken into account that each DWDM channel is divided into frequency slots and the proposed cost-effective ROADM switches all frequency slots of a DWDM channel in a same direction, as described in the Section II. We shall also prove that to reach that maximum throughput value it is not necessary that all cost-effective ROADMs of the network present the colorless feature. That also means a reduction of the implementation cost and the insertion loss (Eq. 1-4) of some of the cost-effective ROADMs of the network. For that purpose, a network simulator has been implemented in MATLAB ${ }^{\mathrm{TM}}$.

\section{A. Scenario details and assumptions}

The Metro-Access Network shown in Fig. 8 has been considered as a reference scenario. It consists of one OMCN, five OANs, four ROADMs and a Passive Optical Networks (PON) connected to each ROADM. The ONUs numbers connected to the different PONs are 4, 8, 16 and 32.

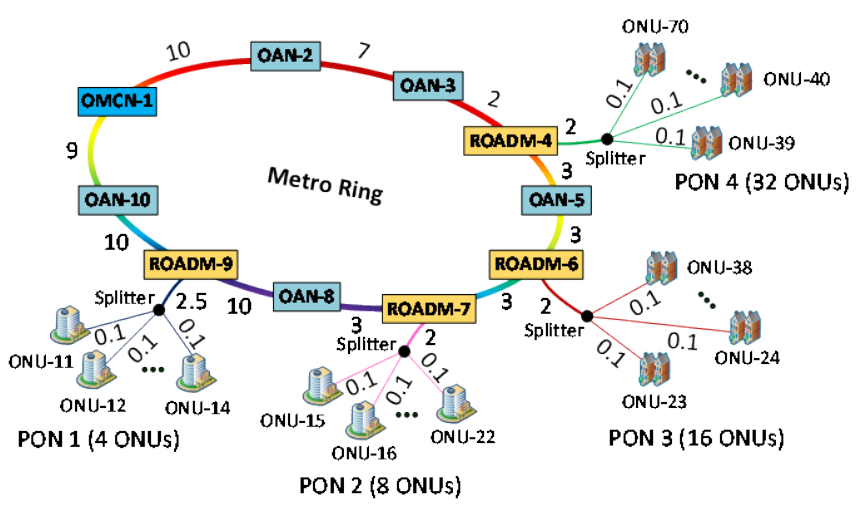

Fig. 8. Network topology considered (link distances are shown in km).

For the sake of simplicity, we considered that the OMCN, OAN and ONU subsystems are able to add/remove specific channels to/from the network to which they are connected. In [6], we proposed a set of different cost-effective solutions for transmitters and receivers and their main attributes for those opto-electrical subsystems implementation. In addition, it is indicated that all those transmitters and receivers can be adapted to implement different u-DWDM transmission techniques in which the up-link and down-link of each user can be established in each FS by frequency or polarization division multiplexing. We select the pair of transmitter and receiver which provides the highest power budget for all opto-electrical subsystems implementation. Following such premise, we consider that the OMCN and OAN, which are usually more expensive equipment, are implemented by Mach-Zehnder transmitters able to provide higher spectral efficient modulation formats as NyquistDPSK. On the other hand, ONU transmitters, much more cost sensitive, could be based on either Distributed Feedback Lasers (DFB) or much lower cost Vertical Cavity Surface Emitting Lasers (VCSEL). For the latter, it has been recently demonstrated the support of DPSK modulation [30] and potentially providing similar spectrum reduced Nyquist-DPSK modulation. The chosen receivers are based on a coherent receiver in which the detected signal is mixed with a local oscillator. As a reference, a DFB local oscillator has been considered, providing a sensitivity of $-52 \mathrm{dBm}[6,10]$. Therefore, the power budget is $52 \mathrm{~dB}$ for transmitters providing $0 \mathrm{dBm}$ output.

The power budget is considered to be only consumed by the fiber attenuation $(0.25 \mathrm{~dB} / \mathrm{km}$ is assumed) and the ROADM insertion loss. We suppose that the OMCN, OAN and ONU insertion losses are null due to their OEO conversion. Chromatic Dispersion (CD) effects due to the optical fiber are not considered as the maximum transmission distance at the network is below the theoretical maximum distance of the link, before considering CD effects (e.g. $61 \mathrm{~km}$ for standard ITU G.652 fibres [31]).

A work band of $500 \mathrm{GHz}$ has been considered as the network resource to share between all users. That work band is considered in order to reduce the computational cost. However, results would scale linearly for higher work bands. Three DWDM channel widths have been considered: $62.5 \mathrm{GHz}, 125 \mathrm{GHz}$ and $250 \mathrm{GHz}$. Being $125 \mathrm{GHz}$ the effective bandwidth obtained for a cost-effective ROADM of a single stage in Section III and $62.5 \mathrm{GHz}$ and $250 \mathrm{GHz}$ the half and double of the effective bandwidth characterized, respectively. Each DWDM channel has been divided into frequency slots, with width of $12.5 \mathrm{GHz}$ [32] although other frequency slot widths could be used, such as $6.25 \mathrm{GHz}$ or 5 $\mathrm{GHz}[10,27]$.

The aforementioned network scenario is loaded with traffic demands distributed uniformly between all pairs of source-destination nodes. All demands are of 10Gbps so each accepted demand takes up one FS. For each request, the two physically paths with the lowest losses (in $\mathrm{dB}$ ) have been considered when generating the paths set for allocating every demand. On the other hand, the worst case path $\left(P_{W C}\right)$ is defined as that path between a pair of sourcedestination nodes which presents the highest total attenuation. Let us remind that the total attenuation depends on the insertion losses generated by transmission through the ROADMs (Eq. 1-4), and the fiber losses between the pair of source-destination nodes.

\section{B. Cost-effective DWDM ROADM design iterative process}

In our study, two important aspects must be considered. The first one is that the proposed ROADM switches all frequency slots of a DWDM channel in the same direction through the configuration of the switches of each stage. The second one is that the cost-effective ROADM provides higher switching flexibility and therefore higher network throughput when a higher number of stages is used. Nevertheless, the ROADM insertion loss increases with the number of stages. Hence, a problem to resolve here is to find out the minimum number of stages of each cost-effective ROADM of the network and their switches configuration so as to reach the network throughput maximum value, while keeping the cost-effective ROADM insertion losses as low as possible. In this way, the optimal design solution for a costeffective ROADM can lose the colorless feature but its implementation cost is thus reduced. 
A greedy way to determine the best set-up of each ROADM to support the maximum throughput would be to consider firstly that each cost-effective ROADM is able to commute all the DWDM channels. In this way, the problem is simplified to search for the best set-up of the cost-effective ROADM stage's switches. Then, the useless stages or the stages which are configured to work as pass-through mode have to be removed. However, this technique may dramatically increase the computational complexity.

Alternatively, the following iterative process can be used:

1. A first step involves finding the best switches configuration of each cost-effective ROADM to achieve a throughput maximum value of the proposed network when a different DWDM channel is assigned to each of ROADMs shown in Fig. 8. In case different configurations generate the same network throughput maximum value, the configuration of the ROADMs which presents a smaller value of the $\mathrm{P}_{\mathrm{WC}}$ is selected. Such $\mathrm{P}_{\mathrm{WC}}$ value must always be lower than the considered power budget; otherwise more sophisticated transmission technologies, providing a higher power budget, would be required.

2. For each ROADM, we calculate the utilization factor (UF). This factor is defined as the sum of the number of occupied FS between each pair of I/O ports, that is E-W, $\mathrm{E}-\mathrm{A} / \mathrm{D}$, and $\mathrm{W}-\mathrm{A} / \mathrm{D}$.

3. In this step, it is decided which ROADM is going to be upgraded to explore potential increase of the network's throughput. The considered upgrading rule is adding extra stages to the ROADM which presents the minimum UF value. If the ROADM with minimum UF is the same as the ROADM of the previous iteration, we select the ROADM with the next higher UF minimum value to the current ROADM. If different ROADMs have the same UF, we select one of them randomly.

4. After the selection of the ROADM to be upgraded, ROADM $_{x}$, it is determined the number of stages, $N_{x}$, for upgrading $\operatorname{ROADM}_{\mathrm{x}}$. For that purpose, the next equation can be used:

$$
N_{x}=\min \left\{\frac{\frac{N_{C} * B W_{C}}{B W_{F S}}-U F_{x}}{\left(B W_{C} / B W_{F S}\right)}, \frac{\left(\frac{A R}{100}-\frac{N D S_{P O N_{x}}}{\frac{N_{C} * B W_{C}}{B W_{F S}}}\right) * N D_{P O N_{x}}}{\left(B W_{C} / B W_{F S}\right)}\right\}
$$

where

- $\quad N_{C}$ is the number of the DWDM channels of the network.

- $\quad B W_{C}$ is the bandwidth of the DWDM channel. It coincides with the effective BW of each ROADM stage.

- $B W_{F S}$ is the bandwidth of each frequency slot.

- $U F_{x}$ is the previous defined utilization factor of the $R O A D M_{x}$.

- $A R$ is a percentage that determine the amount of resources which can be assignment to each PON.

- $N D_{P O N_{x}}$ is the total number of demanded frequency slots that present origin or destination in a node belonging to the PON connected to $R O A D M_{x}$.

- $N D S_{P_{O N N_{x}}}$ is the number of frequency slots of $N D_{P O N_{x}}$ which have been allocated until the current iteration.

The first term of Eq. (5) represents the potential maximum stages number taking into account the number of frequency slots that are available. The second one allows determining the stages number as a function of the resources assigned to each PON. It opens the possibility to assign different priorities for the PONs. The minimum of both terms has to be selected because there might not be enough allowed frequency slots to establish the priority required. This situation takes place when throughput of the network is close to the achievable maximum. If $\mathrm{N}_{\mathrm{x}}$ value is a non-integer, it is rounded to the nearest integer.

5. For determining which DWDM channels can be allocated to $\operatorname{ROADM}_{\mathrm{x}}$, we select those DWDM channels whose frequency slots are not associated with any demand for the different pair of the ROADM I/O ports.

6. For the added stages, it is searched the best switches configuration in order to reach a new maximum throughput value, keeping the switches configuration of previous ROADM's stages unchanged.

7. This process is iterated from step two until all ROADMs have been treated.

\section{Simulation results and discussion}

First, we will evaluate the considered network scenario (Fig. 8), in which the work band of $500 \mathrm{GHz}$ is divided into four DWDM channels of $125 \mathrm{GHz}$. For this situation, two cases will be discussed. The first one is when a different channel is assigned to each cost-effective ROADM. The second one consists of searching for the best configuration of each cost-effective ROADM to reach the maximum throughput following the iterative process detailed previously. Both of them will be compared to the reference case in which the ROADMs are based on WSS and they can switch each frequency slot individually. After this, we will discuss the throughput maximum value behaviour when the network DWDM channel width is changed.

An important aspect to study in the management of the network resources is how they are assigned. A way to allocate those channels is to assign one to each PON without repetition. In this way, all users of the same PON share the same DWDM channel. This strategy coincides with the first step of the iterative process proposed previously and it is the simplest solution in the design of a Metro Ring Network based on cost-effective DWDM ROADMs. Starting with that solution and using the iterative process, a better solution can be achieved. In this situation, different PONs can share the same DWDM channels.

Table IV shows the throughput maximum value of the proposed network in three different cases: i) the Reference Case in which the ROADMs are based on WSS and they can switch each frequency slot individually without any constraint, ii) Case A that corresponds to the situation wherein the cost-effective ROADMs commute all the FS of a DWDM channel in the same direction and a different 
DWDM channel is assigned to each cost-effective ROADM, and iii) Case $B$ that is the result of using the iterative process to reach the throughput maximum value when costeffective ROADMs are applied. For each case, the worst case path, $\mathrm{P}_{\mathrm{WC}}$, and the percentage of allocated traffic demand, $\Delta$, are also reported. That allocated traffic demand percentages is measured against the maximum throughput value of the reference case. From Table IV, it can be deduced that reusing DWDM channel for different PONs the allocated traffic demand can be increased up to $79 \%$ of the reference case whilst in case A the allocated traffic demand is $53.6 \%$. In addition, the worst case path of case B is $43.1 \mathrm{~dB}$, well under the considered power budget of $52 \mathrm{~dB}$.

TABLE IV

THE WORST CASE PATH, THROUGHPUT AND ALLOCATED TRAFFIC DEMAND FOR THE REFERENCE CASE, CASE A AND CASE B

\begin{tabular}{|c|c|c|c|}
\hline \hline Parameter & $\begin{array}{c}\text { Reference } \\
\text { Case }\end{array}$ & Case A & Case B \\
\hline$P_{W C}(\mathrm{~dB})$ & 32.3 & 35.8 & 43.1 \\
\hline Throughput(Gbps) & 1380 & 740 & 1090 \\
\hline$\Delta(\%)$ & 100 & 53.6 & 79 \\
\hline \hline
\end{tabular}

Table $\mathrm{V}$ shows the DWDM channel assigned to each ROADM in the case $B$. That assignment is the result of using the iterative process for some assigned AR. In particular $100 \%, 50 \%, 25 \%$ and $25 \%$ to the PONs connected to ROADM-4, ROADM-6, ROADM-7 and ROADM-9, respectively is considered.

TABLE V

ROADMS CONFIGURATION FOR CASE B (GREY COLOR IS AN ASSIGNED CHANNEL TO THE ROADMX).

\begin{tabular}{|c|c|c|c|c|c|c|}
\hline \hline \multirow{2}{*}{ ROADMx } & \multirow{2}{*}{ AR(\%) } & \multirow{2}{*}{$\begin{array}{c}\text { Stages } \\
\text { Number }\end{array}$} & \multicolumn{4}{|c|}{ Channel } \\
\cline { 4 - 7 } & & 4 & 2 & 3 & 4 \\
\hline 4 & 100 & 4 & & & & \\
\hline 6 & 50 & 2 & & & & \\
\hline 7 & 25 & 1 & & & & \\
\hline 9 & 25 & 1 & & & & \\
\hline \hline
\end{tabular}

Finally, Table VI shows the throughput maximum value of the proposed network as a function of the DWDM channel bandwidth $\left(\mathrm{BW}_{\mathrm{C}}\right)$, specifically for bandwidth of $62.5 \mathrm{GHz}$ (Case C), $125 \mathrm{GHz}$ (Case B, previously studied), and $250 \mathrm{GHz}$ (Case D). For the same AR of each PON as in case $\mathrm{B}$, the worst case path $\left(\mathrm{P}_{\mathrm{WC}}\right)$ and the percentage of allocated traffic demand $(\Delta)$ are also specified. For the cases $\mathrm{C}$ and $\mathrm{D}$, the iterative process described in Section IV.B has been applied in order to determine the network's throughput maximum value. Hence, the stages number of each costeffective ROADM shown in Fig. 8 and their assigned channels do not correspond to case B. From Table VI, it is appreciated that the DWDM channel bandwidth is reduced and the allocated traffic demand is higher. It is due to the fact that the number of wasted FS per channel to satisfy the assigned $\mathrm{AR}$ for each $\mathrm{PON}$ is smaller. However, the ROADMs' number of stages is higher, increasing thus the worst case path losses. So a trade-off solution is when the cost-effective ROADM's DWDM channel width is of $125 \mathrm{GHz}$ (Case B).
TABLE VI

THE WORST CASE PATH, THROUGHPUT AND ALLOCATED TRAFFIC DEMAND AS A FUNCTION OF WDMD CHANNEL BANDWIDTH

\begin{tabular}{|c|c|c|c|}
\hline \hline Parameter & Case C & Case B & Case D \\
\hline$B_{W C}(\mathrm{GHz})$ & 62.5 & 125 & 250 \\
\hline$P_{W C}(\mathrm{~dB})$ & 47 & 43.1 & 36 \\
\hline Throughput(Gbps) & 1190 & 1090 & 960 \\
\hline$\Delta(\%)$ & 86.2 & 79 & 69.7 \\
\hline \hline
\end{tabular}

\section{Conclusion}

In this paper, a cost-effective architecture for DWDM ROADM, whose implementation maximum cost value is an order of magnitude lower than a WSS-based ROADM, has been proposed in order to satisfy the future Metro-Access Networks convergence requirements. A spectrum characterization of single stage cost-effective DWDM ROADM has been also done. From this characterization, a general insertion losses model for a $N$ stages cost-effective ROADM has been proposed. To prove the cost-effective DWDM ROADM performance in a metro ring network scenario, a study of network's throughput has been realized. For that scenario, an iterative process to determine the optimal design of the cost-effective DWDM ROADMs of the network has been presented. Using that process, the allocated traffic is of $80 \%$ to the total traffic that a network using WSS-based ROADMs with FS granularity. We have also demonstrated that the trade-off solution for different DWDM channel widths is of $125 \mathrm{GHz}$ which corresponds to the effective band of the single stage cost-effective ROADM characterized here.

\section{ACKNOWLEDGMENT}

This work was supported in part by SUNSET (TEC201459583-C2-1-R) co-funded by FEDER, the Diputación General de Aragón under grant T25, MECD grant FPU13/00620, MINECO FPI-BES-2015-074302 and, Centro Universitario de la Defensa project BIO-DIVING (CUD2016-18).

\section{REFERENCES}

[1] M. Fiorani, et al., "Challenges for 5G transport networks," in Proc. ANTS, New Delhi. India, pp. 1-6, Dec. 2014.

[2] H. Song, B. Kim, and B. Mukherjee, "Long-reach optical access networks: a survey of research challenges, demonstrations, and bandwidth assignment mechanisms," IEEE Comm. Surv. \& Tutor. 12(1), 112-123, 2010.

[3] J. A. Lazaro, J. Prat, P. Chanclou, G. M. Tosi Beleffi, A. Teixeira, I. Tomkos, R. Soila, and V. Koratzinos, "Scalable extended reach PON," in Optical Fiber Communication Conference/National Fiber Optic Engineers Conference, OSA Technical Digest (CD) (Optical Society of America, 2008), paper OThL2.

[4] H. Rohde, et al., "Coherent Ultra Dense WDM technology for Next Generation Optical Metro and access Networks," J. Lightw. Technol., vol. 32, no. 10, pp. 2041-2052, 2014.

[5] Aleksic, Slavisa. "Towards fifth-generation (5G) optical transport networks," in 2015 17th International Conference on Transparent Optical Networks (ICTON). IEEE, 2015.

[6] Sarmiento, S., Montero, R., Altabas, J. A., Izquierdo, D., Agraz, F., Pagès, A., ... \& Garces, I. "SDN-enabled flexible optical node designs and transceivers for sustainable metro-access 
networks convergence," in 2016 18th International Conference on Transparent Optical Networks (ICTON), IEEE, 2016.

[7] LI, Yongcheng, et al. "Impact of ROADM colorless, directionless, and contentionless (CDC) features on optical network performance [invited]," Journal of Optical Communications and Networking, 2012, vol. 4, no 11, p. B58B67.

[8] Collings, Brandon. "New devices enabling software-defined optical networks." IEEE Communications Magazine 51.3 (2013): 66-71.

[9] Gringeri, S., Basch, B., Shukla, V., Egorov, R., \& Xia, T. J. (2010). "Flexible architectures for optical transport nodes and networks," IEEE Communications Magazine, 48(7), 40-50.

[10] J.A. Altabas, David Izquierdo, J.A. Lazaro, A. Lerin, F. Sotelo, and I. Garces, "1Gbps full-duplex links for ultra-dense-WDM $6.25 \mathrm{GHz}$ frequency slots in optical metro-access networks," OPTICS EXPRESS, Vol. 24, Issue 1, pp. 555-565, 2016.

[11] "1x2 Flexible-grid Wavelength Selective Switch WSS-100." www.santec.com.

[12] "1x2 Flexgrid Wavelength Selective Switch (WSS)." www.finisar.com.

[13] Rambach, Franz, et al. "A multilayer cost model for metro/core networks." Journal of Optical Communications and Networking 5.3 (2013): 210-225.

[14] "Passive Optical Networks Components." www.fs.com.

[15] "Passive Optical Networks Components." www.oequest.com.

[16] Marom, Dan M., et al. "Survey of photonic switching architectures and technologies in support of spatially and spectrally flexible optical networking." Journal of Optical Communications and Networking 9.1 (2017): 1-26.

[17] "Arrayed Waveguide Grating (AWG)." www.nttelectronics.com.

[18] Tripathi, Devendra Kr, et al. "Reconfigurable optical add drop multiplexers A Review." Electrical and Computer Engineering: An International Journal 3 (2014).

[19] Eldada, Louay. "Advances in ROADM technologies and subsystems." Photonics North 2005. International Society for Optics and Photonics, 2005.

[20] JDS Uniphase, "WDM, Filter, $200 \mathrm{GHz}$, ITU Component DWS Series," www.oequest.com/getproduct/18257/cat/1633/page/1.

[21] "1x32 PLC Fiber Splitter, Splice/Pigtailed ABS Module, 3.0mm, SC/APC.” http://www.fs.com/products/48464.html.

[22] "1x2 Opto-Mechanical Optical Switches." http://www.fs.com/products/14376.html.

[23] "2x2 Opto-Mechanical Optical Switches." http://www.fs.com/products/14380.html.

[24] " ROADM in the next generation network." Optelia's white paper, 2013.

[25] Nesset, Derek. "NG-PON2 technology and standards." Journal of Lightwave Technology 33.5 (2015): 1136-1143.

[26] "24-Channel, C-Band $100 \mathrm{GHz}$ Multiplexer." www.oequest.com/getproduct/18697/cat/1337/page/1.

[27] Altabas, Jose A., et al. "1Gbps full-duplex $5 \mathrm{GHz}$ frequency slots uDWDM flexible Metro/Access Networks based on VCSEL-RSOA transceiver," OptoElectronics and Communications Conference (OECC) held jointly with 2016 International Conference on Photonics in Switching (PS), 2016 21st. IEEE, 2016.

[28] Glance, B. "Polarization independent coherent optical receiver." Journal of Lightwave Technology 5.2 (1987): 274276.

[29] ITU-T Recommendation, G.975.1 (2004).

[30] J.A. Altabas, David Izquierdo, J.A. Lazaro, and I. Garces, "1.25-2.5Gbps Cost-Effective Transceiver Based on Directly Phase Modulated VCSEL for Flexible Access Networks," OFC, 2017.

[31] Chauvel, Gildas. "Dispersion in Optical Fibers," Anritsu Corp, 2008.

[32] ITU-T Recommendation, G.694.1 (2012). 